\title{
Berufliche Weiterbildung für Militärärztinnen und Militärärzte
}

\section{Martin Oberholzer}

Prof. Dr. med. Dr. h. c., Dekan der SAMK
Korrespondenz:

LBA - Sanität

Geschäftsstelle SAMK

Worblentalstrasse 36

CH-3063 Ittigen

Tel. 0313242760

Fax 0313237197

info-samk@vtg.admin.ch
Die Schweizerische Akademie für Katastrophen- und Militärmedizin (SAMK) ist zu einer festen Institution der Armee und des Koordinierten Sanitätsdienstes (KSD) geworden. Auch in diesem Jahr wird den Militärärztinnen und Militärärzten durch die SAMK ein breites Angebot an Weiterbildungskursen und Fachtagungen angeboten, die im Rahmen der Dienstpflicht individuell und als besoldete Dienstleistung besucht werden können. Angeboten werden sowohl Fachdienstkurse der Armee (FDK) als auch Fachtagungen und Kurse verschiedener Fachgesellschaften, deren Besuch durch die SAMK finanziert wird. Darunter sind auch viele Kurse, deren Absolvierung für die Erlangung verschiedener Facharzttitel gefordert wird.

\section{Kursangebote der SAMK / Offres des cours ASMC 2011}

* = bereits ausgebucht/occupés

Anästhesie und Reanimation in Basel

Kurssprache deutsch

Anästhesiekongress (englisch)

8./ 9. 4.2011

BS 1

Dienstarztkurs (DAK)

$25 . / 26.8+12 . / 13.9 .2011$ BS 2

ACLS (Advanced Cardiovascular Life Support)*

5./6.5.2011

BS 3

ATLS (Advanced Trauma Life Support)*

29.-31.8.2011

BS 4

PALS (Pediatric Advanced Life Support)*

15./16.9.2011

BS 5

TPAM (Training in Prehospital Airway Management)*

29.6.2011

BS 6

TPAM (Training in Prehospital Airway Management)*

8.11 .2011

BS 7

Sanitätsdienstliche Führung Grossereignis (SFG)

(Teil A: «Knowledge», Grundlagen, Prinzipien, Partner.

Voraussetzungen: Notarzt SGNOR oder Rettungssanitäter

in Kaderposition (oder äquivalent). (deutsch)

Sanitätsdienstliche Führung Grossereignis (SFG)

(Teil B: «Training», Kommunikation und

Teaminteraktion), (deutsch). Voraussetzung: SFG-A

Innere Medizin, Infektiologie in Bern

33. Infektiologie-Symposium, Luzern

24.3.2011

BE 1A

4. Kombiniertes Infektiologie-Curriculum und -Symposium

10.11.2011

BE 1B

10. Berner Notfall-Symposium

20.10.2011

BE $2 A$

6. Systematisches Curriculum, internmedizinische

21.10 .2011

BE 2B

Notfallmedizin

ACLS Kurs (Bern)*

21./22.6.2011

BE 3

ACLS Kurs (Bern)*

23./24.6.2011

ACLS Kurs (Bern)*

23./24.8.2011

25./26.8.2011

25./26.8.2011

19./20.5. + 16./17.6.2011

$25 . / 26.8 .+12 . / 13.9 .2011$

29./30.9.2011

S SFG-B

urs (Bern)

Dienstarztkurs SGNOR, Zürich*

Dienstarztkurs SGNOR, Basel

1. SGIM Great Update für Grundversorger
BE 4

BE 5

BE 6

BE 7

BE 8

BE 9

BE10

Herbstkongress der SGIM 


\section{Kursangebote der SAMK / Offres des cours ASMC 2011}

* = bereits ausgebucht/occupés

Médecine et management en cas de catastrophe et de guerre, à Lausanne

Cours en langue française

Principes de médecine de catastrophe*

27./28.1.2011

VD 1 [M1]

Accident majeur, cours pour professionnels de la chaîne des urgences

23.-25.6.2011

VD $2[\mathrm{M} 2]$

Accident majeur, cours de conduite pour médecins-chefs de secours

6./7.10.2011

VD $3[\mathrm{M} 3]$

et ambulanciers chef des secours*

Plan catastrophe et gestion de crise à l'hôpital

29./30.9.2011

VD $6[\mathrm{M} 6]$

Formation pré-requise: VD 1 [M1]

Cours de médicine d'urgence (SSMUS)

15.-18.11.2011

VD 7

ACLS (Advanced Cardiovascular Life Support)*

17./18.5.2011

VD 8

ACLS (Advanced Cardiovascular Life Support)*

19./20.5.2011

VD 9

ACLS (Advanced Cardiovascular Life Support)*

8./9.11.2011

VD 10

PALS (Pediatric Advanced Life Support)*

23./24.5.2011

VD 11

ATLS (Advanced Trauma Life Support)*

5.-7.9.2011

VD 12

\section{Chirurgie à Genève}

Cours de chirurgie de guerre et de catastrophe

29./30.9.2011

GE 1

Cours de base de suture

10.3.2011

GE 2

Les bases de la laparoscopie

12.5.2011

GE 3

Suture mécanique et laparoscopique

17.8.2011

GE 4

Katastrophen- und Wehrpsychiatrie in Zürich

**Die Kurse ZH 3 bis ZH 10 können nur im Paket zusammen mit den Kursen ZH 1 oder ZH 2 gebucht werden

Kernkompetenz Stressmanagement:

7./8.4.2011

Umgang mit Belastungen in der Akutmedizin.

Kernkompetenz Stressmanagement:

15./16.9.2011

ZH 2

Umgang mit Belastungen in der Akutmedizin.

ACLS (Advanced Cardiovascular Life Support)*

30.6./1.7.2011

$\mathrm{ZH} 3^{* *}$

ACLS (Advanced Cardiovascular Life Support)*

25./26.8.2011

$\mathrm{ZH} 4^{* *}$

ACLS (Advanced Cardiovascular Life Support)

24./25.11.2011

$\mathrm{ZH} 5^{\text {** }}$

ATLS (Advanced Trauma Life Support)*

16./17.6.2011

$\mathrm{ZH} 6^{* *}$

ATLS (Advanced Trauma Life Support)*

20./21.6.2011

$\mathrm{ZH} 7^{* *}$

ATLS (Advanced Trauma Life Support)*

26./27.9.2011

$\mathrm{ZH} 8^{* *}$

PALS (Pediatric Advanced Life Support)*

10./11.11.2011

$\mathrm{ZH} 10^{* *}$

Fachzentrum für Zahnmedizin und Kieferchirurgie, Basel

SAKM (Schweizerische Arbeitsgemeinschaft für

6. Bernd Spiessl Symposium für innovative und

visionäre Technologien

KFO/MKG: Interdisziplinäre Planung und Therapie

3.3.2011

ZM 3

von Dysgnathiepatienten

KFO/MKG: Transversale Problemstellungen

4.-6.03.2011

ZM 4

KFO/MKG: Asymmetrien

7. -9.10 .2011$

\section{Fachzentrum für Transfusionswesen}

13. Weiterbildungstagung und Symposium BSD SRK/SVTM in Interlaken

offen

TW 1

Fachzentrum Medizinische B-Abwehr, Zürich

Emerging Infections

9.6.2011

MBA 1

Kursanmeldungen ausschliesslich elektronisch ab Homepage!

www.lba.admin.ch/internet/lba/de/home/themen/sanit/neu/neu2/inscr2011.html 